\title{
Digit ratio, personality and emotions in skydivers
}

\author{
SIMONA MASSIMINO ${ }^{1}$, SERGIO RINELLA ${ }^{1}$, ANDREA BUSCEMI ${ }^{2,4}$, ELISA SIMILIA $^{1}$, \\ VINCENZO PERCIAVALLE ${ }^{1}$, VALENTINA PERCIAVALLE ${ }^{3}$, MARIA CRISTINA PETRALIA ${ }^{1,4}$, \\ DONATELLA DI CORRADO ${ }^{5}$, ANNARITA LASPINA ${ }^{3}$ and MARINELLA COCO ${ }^{1}$ \\ ${ }^{1}$ Department of Biomedical and Biotechnological Sciences, University of Catania, I-95123 Catania; \\ ${ }^{2}$ Department of Research, Horus Social Cooperative, I-97100 Ragusa; ${ }^{3}$ Department of Educational Sciences; \\ ${ }^{4}$ Department of Research, Italian Center Studies of Osteopathy, I-95100 Catania; ${ }^{5}$ Department of \\ Human and Social Sciences, School of Sport Sciences, Kore University, I-94100 Enna, Italy
}

Received November 23, 2017; Accepted October 24, 2018

DOI: $10.3892 / b r .2018 .1174$

\begin{abstract}
The aim of the present study was investigate if there is an association between second-to-fourth digit length (2D:4D) ratio and personality factors capable of serving as predictors of individual choice towards high-risk activities in a group of experts skydivers; Furthermore, their skills in regulating anxiety and emotions were assessed. The 2D:4D ratio of the right hand of 41 expert skydivers was measured and each of them completed four questionnaires: Big Five Questionnaire-2 (BFQ-2), Profile of Mood States (POMS), State-Trait Anxiety Inventory Form Y (STAI-Y) and Risk-Taking Inventory. Lower 2D:4D ratios did not appear associated with a greater propensity for taking risks but rather with a lower aptitude to assume precautions in unsafe conditions. In fact, the only sub-dimensions of personality, analyzed by the BFQ-2, correlated with the $2 \mathrm{D}: 4 \mathrm{D}$ ratio were conscientiousness and agreeableness. Furthermore, prior to launch, the skydiver's level of stress, measured by the POMS, or state anxiety, measured by the STAI-Y, was not significantly correlated with 2D:4D ratio; whereas there was significant positive correlation between 2D:4D values and trait anxiety. Data analysis further revealed that social desirability correlated negatively with state anxiety and total mood disturbance index, and positively with emotion control. The present results suggest that lower 2D:4D ratio may represent a significant predictor of less attentive precautionary behavior when risk-taking.
\end{abstract}

Correspondence to: Dr Marinella Coco, Department of Biomedical and Biotechnological Sciences, University of Catania, 89 S. Sofia, I-95123 Catania, Italy

E-mail: marinella.coco@unict.it

Dr Andrea Buscemi, Department of Research, Italian Center Studies of Osteopathy, 1 Marziano, I-95100 Catania, Italy

E-mail: andreabuscemi@virgilio.it

Key words: second-to-fourth digit length ratio, risk-taking, Big Five Questionnaire-2, mood state, anxiety

\section{Introduction}

Skydiving is classified among other high-risk sports, which are generally defined as activities in which the individual accepts the possibility of personal injury or fatality (1-2). Given the potential risks associated with high-risk behaviors, it is interesting to identify the distinctive features in the personality of individuals who engage in these activities.

Zuckerman (3) observed that certain individuals are less able to tolerate monotonous situations compared with others. These subjects become immediately agitated and experience a feeling of irritation when not stimulated. It was suggested that the emerged differences in this experimental situation derived from a primary behavioral disposition (3). Zuckerman further identified a personality trait, termed sensation-seeking, as 'a trait defined by the seeking of varied, novel, complex and intense sensations and experiences, and the willingness to take physical, social, legal and financial risks for the sake of such experience' $(4,5)$.

Therefore, the personality of the individual is an important predictor of such a behavior; specifically, two factors of the Big Five Theory (6,7), extraversion and conscientiousness, are the most studied in the context of risk-taking (8-11).

Previous research identified that individuals who deliberately engaged in activities associated with stress and danger were able to better regulate anxiety and emotion than others who avoided the high-risk stressful situations $(12,13)$. Through long-term practice of high-risk activities, participants may learn strategies of self-regulation from the high-risk experience and, hypothetically, transfer them to everyday life situations (14)

Skydiving is a natural stressor from a physiological and psychological point of view. Previous reports have highlighted a difference between experts and beginners in the capability of regulating anxiety $(15,16)$. Hare et al $(15)$ assessed the cortisol levels and self-reported anxiety intensity in relation to emotional changes pre- and post-jump. They identified that post-jump cortisol levels post-jump were similarly high in experts as in beginners, but the intensity of self-reported anxiety was lower in the experts.

Other studies have suggested that prenatal androgens affect the developing brain by increasing its sensitivity to circulating 
testosterone later in life (17-19). These effects may include increased self-confidence (20), search persistence and risk preferences (21-23), and intensified vigilance and quickened reaction times $(24,25)$. A number of markers have been proposed for evaluating the effects of prenatal androgens (26), though the most suitable is considered to be the second-to-fourth digit length (2D:4D) ratio (27), with a relatively longer fourth finger (lower 2D:4D ratio) indicating higher fetal androgen levels (28). However, a previous study concluded that, due to the considerable within-group variability and between-group overlap, digit ratio is not a suitable marker of individual differences in prenatal androgen exposure (29). Recently, Manning (30) suggested that 2D:4D is determined not by prenatal androgens alone but by the balance of prenatal androgen to prenatal estrogen signaling in a narrow time window of fetal digit development. For ease of measurement and reproducibility, 2D:4D may be used as a substitute measure for prenatal androgen exposure (29-32). In fact, 2D:4D ratios appear to be correlated with certain personological factors (neuroticism, agreeableness, openness) $(33,34)$ that appear to be predictive of success among high-frequency financial traders $(35,36)$, of acceptance into and success in medical schools of state-run Italian universities (37), of performance in competitive sports, including basketball (38), skiing and soccer $(39,40)$, and of aggressive behavior in professional soccer players $(41,42)$; in general, it appears to be predictive of risky decision making (43).

In the present study personality factors were assessed in expert skydivers following the Big Five Theory (6,7). The Big Five Questionnaire-2 (BFQ-2) was used, which also includes, through a lie scale, the evaluation of social desirability (44). This scale measures the tendency in the subject to provide a false 'positive' or 'negative' profile of themselves. Social desirability is the tendency of an individual to give untruthful answers, in order to present their self in a favorable light and to demonstrate their suitability to the social environment, which is indicative of tolerant, open-minded, rational, democratic and unprejudiced behaviors (45).

The capability to control emotions, anxiety and mood was also assessed using the State-Trait Anxiety Inventory Form Y (STAI-Y) (46) and the Profile of Mood States (POMS) (47).

Woodman et al (48) validated a measure that allows researchers to assess risk-taking in high risk-sport athletes; they validated the Risk Taking Inventory (RTI) for high-risk sport, by postulating a dichotomy of risk-taking behavior, deliberate risk-taking (DRT) versus precautionary behavior (PB), that may be utilized for evaluating the propensity to take risks and tendency to assume PBs.

The aim of the present study was to assess the personological features of a group of expert skydivers, as well as to investigate their ability to regulate anxiety and mood state, and to evaluate if these abilities are associated with 2D:4D ratio; furthermore, it was investigated if there is an association between 2D:4D ratio and personality factors capable of serving as predictors of individual choice towards high-risk activities, such as extraversion and conscientiousness $(33,34,42,43)$.

\section{Subjects and methods}

Participants. Members of the sport associations 'SunflyersParacadutismo sportivo' and 'Skydive Sicilia', enrolled at UIP
(Italian Union Parachuting) were enrolled in the current study between May and June, 2016. Volunteers provided written informed consent to the study procedures that were conducted in accordance with the Declaration of Helsinki. The subjects were also informed on their right to privacy and anonymity and the study was approved by the Ethical Committee of Kore University (Enna, Italy).

The sample consisted of 41 healthy subjects, aged between 18 and 46 years [mean \pm standard deviation (SD), 31.73 \pm 5.51 ] and included 24 males (mean age, $31.83 \pm 6.84$ years) and 17 females (mean age, $31.56 \pm 2.94$ years).

The participants belonged to groups of expert skydivers based in Sicily, Italy; a threshold was adopted to distinguish experts from beginners as those who had been practicing the activity for a minimum of 12 consecutive months. The observed subjects had been practicing the sport for a period of 12 months minimum to a maximum of 228 months (mean time, $37.8 \pm 38.1$ months). The subjects were distributed by practice time as follows: 12 to 24 months $(n=11) ; 25$ to 36 months $(n=12)$; 37 to 72 months $(n=15) ;>72$ months $(n=3)$. The frequency of annual jumps ranged from 4 to 70 (mean frequency, 19.1 14.1 ), distributed as follows: 4 to 10 jumps $(n=7) ; 11$ to 20 jumps $(n=20) ; 21$ to 30 jumps $(n=4) ; 31$ to 40 jumps $(n=7) ;>41$ jumps $(n=3)$.

Subjects excluded from the study were those who i) had $<12$ consecutive months experience; and ii) obtained a standardized score (T-score) $\geq 65$ on the lie scale and at least on 3 major factors of the BFQ-2. The latter criterion is indicated by the authors of the BFQ-2 to identify the falsified personological profiles that should be excluded since they present results that are not reliable (44).

Procedures and measures. To perform the study, 2D:4D ratio was measured and psychological assessment tests were performed, including BFQ-2, POMS, STAI-Y and RTI, at $2 \mathrm{~h}$ prior to launch.

Digit ratio measurement. The method used for measuring the 2D:4D ratio was described by our group in a previous study (37). Briefly, the participants' right hands were photocopied and the digit length from the metacarpophalangeal crease to the fingertip was measured. It has been reported that this crease appears around the 9th week of gestation and is one of the primary creases of the hand (49). The 2D:4D ratio was determined from only the right hand, as the right-hand digit ratios appear to be more sensitive to prenatal androgens $(50,51)$. The handprints of the subjects were measured to determine 2D:4D ratio using calipers accurate to $0.1 \mathrm{~mm}$. The lengths of each participant's fingers were measured twice, and the mean was calculated.

$B F Q-2$. The BFQ-2 questionnaire by Caprara et al (44) is one of the most used in Italy for measurement of the Big Five personality factors. Construct validity was confirmed by the relationship with other tools proposed for the measurement of personality, including the NEO-Personality Inventory (52). The theoretical reference related to the Costa and McCrae Big Five Theory (6) for all factors was maintained. The scores obtained are divided into very low scores (between 25 and 35), low scores (between 36 and 45), normal scores (between 
46 and 55), high scores (between 56 and 65) and very high scores (between 66 and 75) (44).

The BFQ-2 assesses personality traits divided into 5 major factors, each of which are divided into two sub-dimensions:

Openness (inventive/curious vs. consistent/cautious): The degree of intellectual curiosity, creativity and preference for novelty and variety an individual has; also defined as the extent to which a person is imaginative or independent, and represents a personal preference for a variety of activities over a strict routine. In BFQ-2 it is termed openness and is divided into two sub-dimensions: Openness to culture and Openness to experience.

Conscientiousness (efficient/organized vs. easy-going/ careless): Propensity to be organized and dependable, show self-discipline, act dutifully, aim for success, and prefer planned rather than spontaneous behavior. In BFQ-2 it is divided into two sub-dimensions: Scrupulousness and Perseverance.

Energy (or extraversion; outgoing/energetic vs. solitary/ reserved): Energy, positive emotions, surgency, assertiveness, sociability and the tendency to seek stimulation in the company of others, and talkativeness. In BFQ-2 it is termed energy and is divided into two sub-dimensions: Dynamism and Dominance.

Agreeableness (friendly/compassionate vs. analytical/ detached): Propensity to be empathetic and cooperative rather than suspicious and hostile towards others; it is also a measure of one's trusting and helpful nature, and whether an individual is generally well tempered or not. In BFQ-2 it is termed friendliness and is divided into two sub-dimensions: Cooperativeness and Politeness.

Emotional stability (or neuroticism; sensitive/nervous vs. secure/confident): Propensity to experience unpleasant emotions easily, such as anger, anxiety, depression and vulnerability; it also denotes the degree of emotional stability and impulse control. It is divided into two sub-dimensions: Emotion control and Impulse control.

A sixth, 12-item control scale, termed lie scale, consisting of two sub-dimensions (lie egoistic + lie moralistic), was added. This scale measures the participant's tendency to provide a false profile of him/herself. The items are rated on a 5-point Likert scale ranging from 1 (absolutely false) to 5 (absolutely true).

Costa and McCrae report for BFQ-2 an internal consistency with Cronbach $\alpha$ coefficients ranging from 0.73 to 0.86 (52). The $\alpha$ coefficients for the Italian questionnaire are also relatively high (44); an $\alpha$ of 0.74 was reported for the lie scale.

POMS. POMS provides a measure of mood states (47). Respondents completing the questionnaire rate each item on a 5 -point Likert scale with anchors ranging between 0 (not at all) to 4 (extremely). Internal consistency for this method is reportedly high $(\mathrm{r}=0.90)(47)$. The items are combined to form six separate subscales: Tension-anxiety $(\mathrm{T})$, depression-dejection (D), anger-hostility (A), vigor-activity (V), fatigue-inertia (F) and confusion-bewilderment (C). The six subscale standardized scores (T-scores) are then combined to form an overall measure of affect that is known as total mood disturbance $(\mathrm{TMD}=\mathrm{T}+\mathrm{D}+\mathrm{A}-\mathrm{V}+\mathrm{F}+\mathrm{C})$.

STAI-Y. The STAI-Y is a psychological inventory based on a 4-point Likert scale, 1 (not at all) to 4 (extremely), and consists of 40 questions on a self-report basis (46). The STAI measures two types of anxiety: State anxiety, or anxiety about an event; and Trait anxiety, or anxiety level as a personal characteristic. Higher scores are positively correlated with higher levels of anxiety. Its most current revision is Form Y.

RTI. The RTI evaluates the risk-taking behavior of a subject that lends their self to conduct a high-risk sport (48). The RTI has seven items across two factors: DRT (e.g., he/she actively seeks out dangerous situations) and PB (e.g., he/she takes time to check for potential hazards). Items are rated on a 5-point Likert scale (1, never; 5, always).

Data analysis. Data was collected and averaged, and then compared by using the non-parametric Mann-Whitney test. Linear regression and Pearson's correlation coefficients were also determined. Significance was set at $\mathrm{P}<0.05$. All descriptive statistics are reported as mean \pm SD. All analyses were performed using GraphPad Prism version 5.0 for Windows (GraphPad Software, Inc., La Jolla, CA, USA).

\section{Results}

Subject data. The measurement of 2D:4D ratios was calculated in centimeters $(\mathrm{cm})$ and gave values distributed between 0.911 and $1.028 \mathrm{~cm}$, with a mean of $0.966 \pm 0.028 \mathrm{~cm}$. The difference in 2D:4D ratios between males $(n=24 ; 0.951 \pm 0.018 \mathrm{~cm})$ and females $(\mathrm{n}=17 ; 0.972 \pm 0.032 \mathrm{~cm})$ was not significant $(\mathrm{P}<0.05$; Mann-Whitney test; data not shown). Mean values of the five major factors and the lie scale of the BFQ-2, as well as the mean values of the sub-dimensions of the BFQ-2, are reported in Table I.

Agreeableness had the highest mean score (54.46 \pm 8.56$)$, while conscientiousness had the lowest mean score (50.90 \pm 12.00$)$. Among the sub-dimensions, cooperativeness had the highest mean score $(55.13 \pm 8.44)$, whereas openness to culture had the lowest mean score $(46.55 \pm 9.74)$.

Table II presents the mean values of the 6 factors of the POMS. The factor vigor had the highest mean score (61.68 \pm 9.20$)$, whereas the factor tension had the lowest mean score $(41.88 \pm 2.70)$. The mean value of the TMD index was $152.73 \pm 20.25$.

Regarding the STAI-Y, it was observed for state anxiety a mean value of $35.39 \pm 8.78$ and for trait anxiety a mean value of $36.53 \pm 6.92$. Both values were within the normality range (46) and did not differ statistically (data not shown).

Correlation analysis. Possible correlations between sub-dimensions of the BQF-2, the TMD index of the POMS and the STAI-Y were analyzed, and four significant correlations were detected: i) A positive correlation $\left(\mathrm{R}^{2}=0.3795\right.$; $\mathrm{P}=0.0020$ ) between the sub-dimensions lie egoistic and emotion control of the BFQ-2; ii) a negative correlation $\left(\mathrm{R}^{2}=0.3028 ; \mathrm{P}=0.0063\right)$ between the lie scale of the BFQ-2 and the TMD index of the POMS; iii) a negative correlation $\left(\mathrm{R}^{2}=0.4450 ; \mathrm{P}=0.0003\right)$ between the sub-dimension lie egoistic of BFQ-2 and state anxiety of the STAI-Y; and iv) a negative correlation $\left(\mathrm{R}^{2}=0.4717 ; \mathrm{P}=0.0137\right)$ between the factor vigor of the POMS and state anxiety of the STAI-Y (data not shown). 
Table I. Mean scores of BFQ-2 factors and sub-dimensions.

\begin{tabular}{lcc}
\hline Variables & Mean & Standard deviation \\
\hline BFQ-2 factor & & \\
Energy & 52.37 & 8.77 \\
Agreeableness & 54.46 & 8.56 \\
Conscientiousness & 50.90 & 12.00 \\
Emotional stability & 52.93 & 10.04 \\
Openness & 51.44 & 10.16 \\
Lie scale & 52.02 & 8.77 \\
BFQ-2 sub-dimension & & \\
Dynamism & 54.82 & 8.24 \\
Dominance & 50.88 & 10.04 \\
Cooperativeness & 55.13 & 8.44 \\
Politeness & 54.10 & 9.37 \\
Scrupulousness & 49.15 & 10.89 \\
Perseverance & 53.95 & 12.69 \\
Emotion control & 53.65 & 10.50 \\
Impulse control & 53.30 & 7.98 \\
Openness to culture & 46.55 & 9.74 \\
Openness to experience & 54.75 & 9.94 \\
Lie egoistic & 54.17 & 9.34 \\
Lie moralistic & 50.62 & 7.83 \\
\hline
\end{tabular}

Normative reference: very low score, 25-35; low score, 36-45; normal score, 46-55; high score, 56-65; very high score, 66-75 (44). BFQ-2, Big Five Questionnaire-2.

Table II. Mean values of POMS factors and TMD index.

\begin{tabular}{lcc}
\hline POMS factor & Mean & Standard deviation \\
\hline Tension & 41.88 & 2.70 \\
Depression & 43.32 & 2.65 \\
Anger & 42.85 & 2.90 \\
Vigor & 61.68 & 9.20 \\
Fatigue & 42.56 & 4.49 \\
Confusion & 43.80 & 7.06 \\
TMD & 152.73 & 20.25 \\
\hline
\end{tabular}

Normative reference (expressed as overall mean score in males and females): Tension, 13.4; depression, 13.4; anger, 9.7; vigor, 15.6; fatigue, 10.5; confusion, 10.9 (41). POMS, Profile of Mood States; TMD, total mood disturbance.

No other significant correlations were detected between the STAI-Y and the BFQ-2 except for that for emotional stability and its sub-dimensions. The factor vigor did not exhibit any significant correlations with the factors or sub-dimensions of the BFQ-2.

Fig. 1 illustrates the correlations between 2D:4D and DRT, PB, state anxiety and trait anxiety. A significant positive correlation was observed between $2 \mathrm{D}: 4 \mathrm{D}$ and $\mathrm{PB}\left(\mathrm{R}^{2}=0.2942\right.$; $\mathrm{P}=0.0002)$ as well as between $2 \mathrm{D}: 4 \mathrm{D}$ and trait anxiety
$\left(\mathrm{R}^{2}=0.1791 ; \mathrm{P}=0.0058\right)$. Therefore, skydivers with lower $2 \mathrm{D}: 4 \mathrm{D}$ ratios were indicated to be less careful in taking precautions when deciding to take a risk.

In this respect, it is notable that, although no significant association between 2D:4D and the overall score of the BQF-2 was observed $\left(\mathrm{R}^{2}=0.2386 ; \mathrm{P}=0.1229\right.$; data not shown $)$, the correlation of 2D:4D with individual factors of the BFQ-2, as illustrated in Fig. 2, were significant: There were positive correlations between 2D:4D and the factors conscientiousness $\left(\mathrm{R}^{2}=0.1940 ; \mathrm{P}=0.0039\right)$ and agreeableness $\left(\mathrm{R}^{2}=0.1086\right.$; $\mathrm{P}=0.0353$ ) of the BFQ-2. This implies that individuals with lower 2D:4D ratios are less conscientious.

\section{Discussion}

In the sample of expert skydivers analyzed in the present study, the 2D:4D ratio appeared unrelated to capability to take risk but rather with the aptitude to assume a PB in unsafe conditions. this observation is supported by the finding that the only factors of personality, analyzed by the BFQ-2, that correlated with 2D:4D ratio were conscientiousness and agreeableness. Furthermore, prior to launch, skydivers did not exhibit a significant correlation between their 2D:4D ratio and level of stress, measured by the POMS, or level of state anxiety, assessed by the STAI-Y. However, a significant positive correlation was determined between 2D:4D values and trait anxiety, measured by the STAI-Y. Therefore, it seems reasonable to conclude that subjects with a low 2D:4D ratio may be less conscientious in taking precautions when they decide to take a risk, which is in part supported by the lower trait anxiety observed among the present cohort.

The variable conscientiousness of the BFQ-2, indicating the inclination to be organized and dependable and to prefer planned rather than spontaneous behavior, had the lowest mean score among the five factors. The factor extraversion is a measure of energy as well as agreeableness and willingness for a given activity; it measures aspects including energetic and dynamic behavior, activity, ease of speech, sociability, enthusiasm, and all those aspects related to the ability to influence others. These behavioral patterns appear associated with social desirability, assessed by the BFQ-2 lie scale. The lie scale measures a subject's tendency to provide a false profile of him/herself in order to satisfy the individual desire to belong to a group. To achieve this, the participants invest high levels of energy; this appeared to be demonstrated by the positive correlation between social desirability and the factor energy identified presently.

The factor energy may be another important predictor of risk-taking behavior, particularly for social dynamics (53). As confirmation of this, the mean score of the factor agreeableness was highest among the five factors. This factor is important for the dynamics of a group and is an expression of trust, altruism, compliance, modesty, optimism and loyalty (53).

Furthermore, this finding is supported by the higher scores for cooperativeness (friendly/compassionate), a sub-dimension of the factor agreeableness, compared with the other sub-dimensions.

Thus, one of the reasons that motivates skydivers to engage in high-risk activities may be the desire of the individual to establish meaningful relationships and, therefore, to experi- 

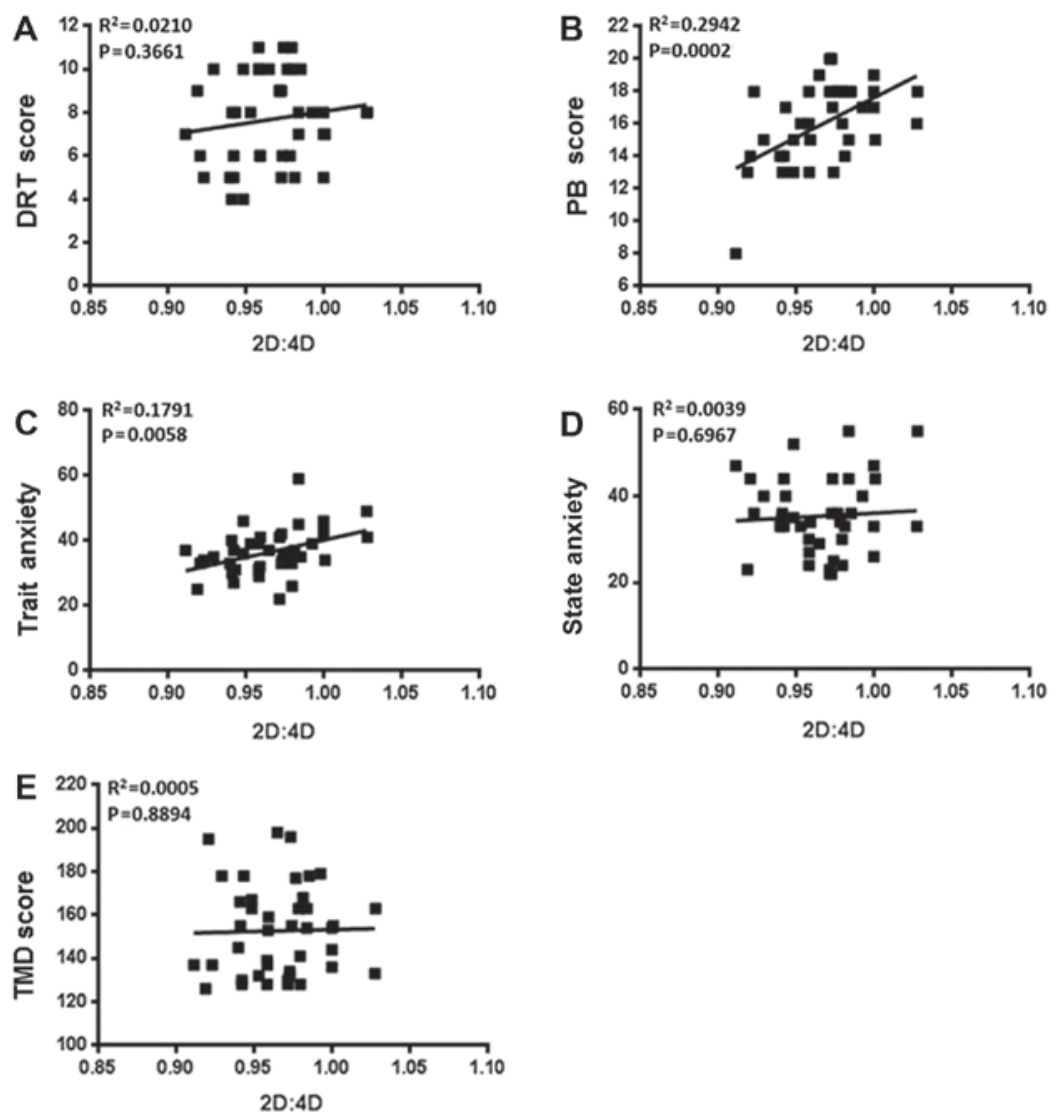

Figure 1. Correlation between 2D:4D and (A) DRT, (B) PB, (C) trait anxiety, (D) state anxiety and (E) TMD. 2D:4D, second-to-fourth digit length ratio; DRT, deliberate risk-taking; $\mathrm{PB}$, precautionary behavior; TMD, total mood disturbance.
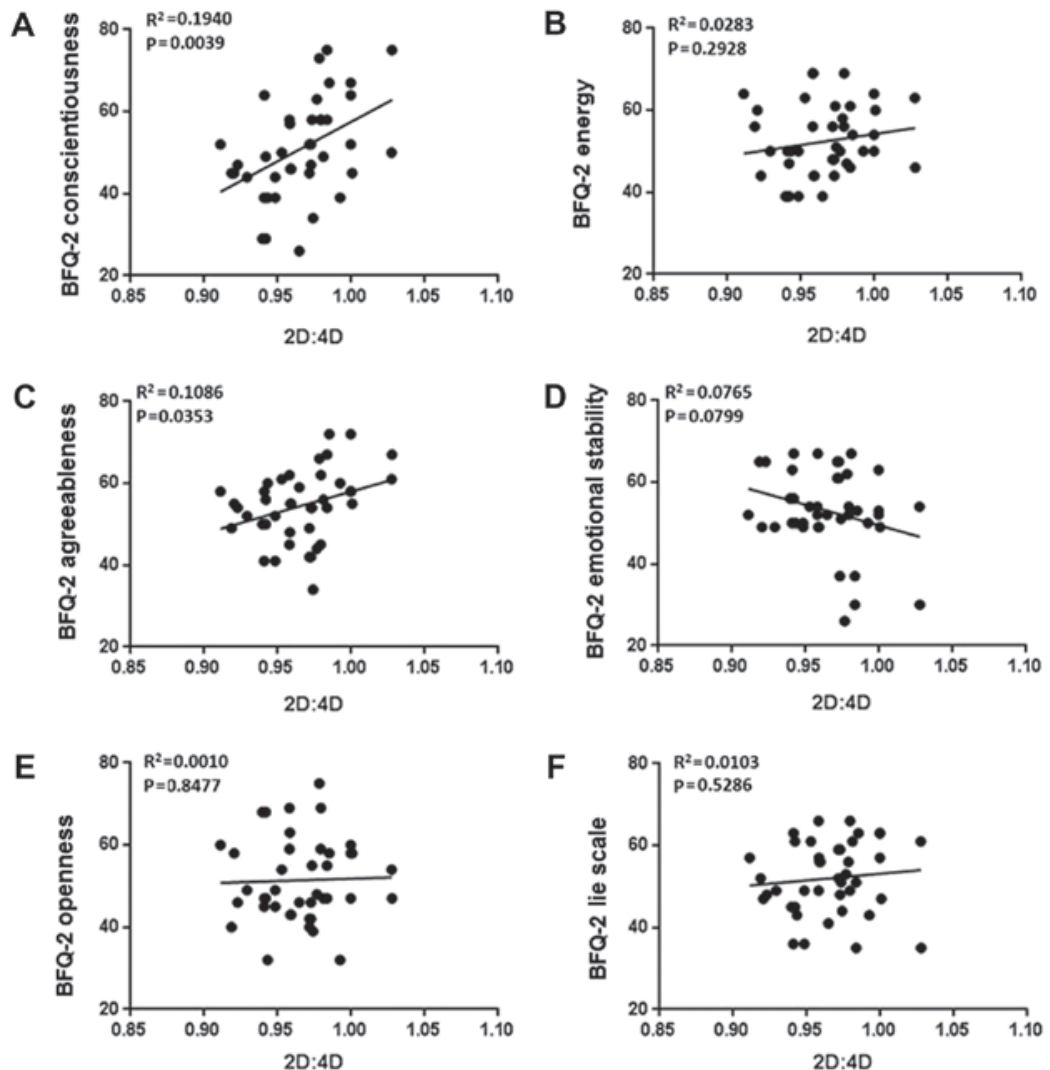

Figure 2. Correlation between 2D:4D and the major factors of the BFQ-2, (A) conscientiousness, (B) energy, (C) agreeableness, (D) emotional stability, (E) openness and (F) lie scale. A significant positive correlation between 2D:4D and the factors conscientiousness and agreeableness. 2D:4D, second-to-fourth digit length ratio; BFQ-2, Big Five Questionnaire-2. 
ence group membership. This would provide a positive reinforcement for risk-taking, obtained by social recognition and by the resulting status of expert (1-54). Ultimately, the present data demonstrate the possible relevance of the group effect, as a theory to support the desire of group membership. Indeed, the present sample of skydivers appeared to invest high levels of energy in order to provide a positive, responsible and courageous profile of him/herself; this appeared useful for maintaining and improving the capacity to control emotional state This is consistent with the positive correlation between the sub-dimension emotion control and the lie egoistic scale (a sub-dimension of the lie scale) of the BFQ-2.

Skydiving involves high emotional investment and high levels of anxiety, particularly pre-jump. Therefore, it is necessary that the individual maintains a positive mood state, useful for efficient control of anxiety and emotions (55). This was indicated by the positive correlation between state anxiety of the STAI-Y, specifically at pre-jump, and the overall mood index, TMD of the POMS. TMD assesses the tendency for mood disorders (56). For participants, social desirability appeared important in terms of mood regulation. Indeed, the TMD index correlated negatively with the lie scale of the BFQ-2, which assesses social desirability, and this appeared capable of positively influencing mood.

The participants, in order to belong to the group, attempted to appear competent, courageous and intelligent; all aspects relating to the personal qualities that are measured by the sub-dimension of the lie scale, lie egoistic. All these aspects, centered on expressing a desire to belong to the group, appeared to facilitate control of state anxiety, mood and emotion. This is consistent with the positive correlation between the factor emotional stability of the BFQ-2, specifically with emotion and impulse control, and social desirability, as emerging from the lie scale of the BFQ-2. When participants exhibited a profile of him/herself as socially desirable, they appeared to maintain good emotional stability, specifically regarding control of emotions and impulse. Thus, the desire of belonging to the group, and the underlying dynamics, appear to be useful for coping with the stress and anxiety of skydiving.

Furthermore, the present investigation confirmed data from previous studies on skydivers, which indicated that a high-risk sport, if practiced for a long time, influences positively the control of anxiety $(15,54,57)$. Participants in those studies, expert skydivers who had practiced the sport for at least four years, seemed to manifest a good control of anxiety and emotion. However, it is conceivable that individuals choosing high-risk activities have good ability to control emotions and anxiety as a personal prerequisite.

It is widely accepted that people who practice high-risk sports in the long-term frequently regulate emotion and anxiety, and in this way reinforce their capability for emotion control (14,58-68). It may be useful to investigate the ways in which individuals learn strategies of self-regulation from a high-risk context, and whether and how they transfer these skills to everyday life situations.

The data analysis demonstrated a significant negative correlation between the factor vigor, a mood state assessed by the POMS, and state anxiety. The factor vigor evaluates a domain of mood characterized by exuberance and energy and is the only factor in negative relationship with the other five factors of the POMS. It seems clear that the factor vigor represents a characteristic of skydiving, useful to support the jump. This is evidenced by the mean score of vigor, which was the highest among the six factors of the POMS. Given the negative correlation between state anxiety and the factor vigor, and its assumed importance for skydiving, it could be argued that practicing skydiving for an extended time may positively affect anxiety control.

Furthermore, the data have indicated the absence of significant correlations between the state and trait anxiety of STAI-Y and the majority of personality factors assessed by the BFQ-2, except for emotional stability, which specifically evaluates emotion and impulse control. The lack of correlation between anxiety and personality traits in the participants suggests that practicing skydiving in the long-term represents a valid tool to control anxiety.

The main goal of the present study was to investigate the personological characteristics that defined the skydivers, and their skills to regulate anxiety and mood state, with the aim of identifying a possible correlation between their $2 \mathrm{D}: 4 \mathrm{D}$ ratio and personological factors; to determine aspects that may serve as predictors of risk-taking and/or PB.

Although the data analysis identified no significant differences in terms of personality traits among the skydivers, the factors conscientiousness and agreeableness, evaluated by the BFQ-2, were positively associated with 2D:4D ratio. The association between 2D:4D ratio and conscientiousness could be considered as a significant predictor of reduced attentiveness in taking appropriate precautions under risk. This possibility is coherent with the positive correlation among skydivers between 2D:4D ratio and $\mathrm{PB}$. The factor energy (or extraversion) of the BFQ-2 may represent a notable variable in this context. Indeed, participants seemed to invest high levels of energy to provide a positive, competent and courageous profile of him/herself, in order to maintain high levels of social desirability (lie scale). Finally, individuals with lower 2D:4D ratios exhibited lower levels of trait anxiety, a factor that may facilitate the choice to take risks.

The present study had some limitations related to sample size. The sample size may not have been sufficient to obtain some of the expected results. This appeared particularly evident for the female parachuting group, which included only 17 participants (male group, $n=24$ ). Collecting a larger sample may allow the acquisition of more robust statistical results. For future studies, a more strategic method should be applied to search for participants. In addition to addressing the shortcomings of the present study, future research may attempt to confirm the present results by studying the association between 2D:4D ratio and personological factors, as well as circulating testosterone and salivary cortisol levels, across a range of individuals who engage in extreme sports and other risk-taking behaviors. Nonetheless, the present work, together with a growing body of similar studies at the hormonal level, demonstrates the importance of biological input in participants' behaviors when considered at risk (69-78).

The present investigation represents the first phase of a larger project that aims to extend investigation to other sports in the domain of risk-taking, in order i) to understand the motivations underlying the choice to practice these sports; and ii) to investigate the ways in which high-risk sports represent 
a possible resource for the control of anxiety, emotions and mood.

\section{Acknowledgements}

Not applicable.

\section{Funding}

No funding was received.

\section{Availability of data and materials}

The datasets generated and/or analyzed during the present study are available from the corresponding author on reasonable request.

\section{Authors' contributions}

MC, SM, VaP and ViP contributed to the conception and design of the study. MC and SM performed the experiments. SM, SR, ES, MC, DDC, AL, VaP, MCP, AB and ViP participated in the analysis and interpretation of the data. $\mathrm{MC}, \mathrm{AB}$ and $\mathrm{VaP}$ contributed to the writing of the article, provided intellectual content and gave approval of the final version of the article to be published.

\section{Ethics approval and consent to participate}

The present study was approved by the Internal Review Board of the Kore University of Enna (Enna, Italy). The subjects provided written informed consent following full disclosure of the study protocol.

\section{Patient consent for publication}

All subjects provided written informed consent permitting publication of relevant results maintaining anonymization of personal information.

\section{Competing interests}

The authors declare that they have no competing interests.

\section{References}

1. Celsi RL, Rose RL and Leigh TW: An exploration of high-risk leisure consumption through skydiving. J Consum Res 20: 1-23, 1993.

2. Pedersen DM: Perceptions of high risk sports. Percept Mot Skills 85: 756-758, 1997.

3. Zuckerman M: Dimensions of sensation seeking. J Consult Clin Psychol 36: 45-52, 1971.

4. Zuckerman M: Behavioral Expressions and Biosocial Bases of Sensation Seeking. Cambridge University Press, New York, NY, 1994.

5. Zuckerman M: Sensation Seeking and risky driving, sports, and vocations. In: Sensation seeking and risky behavior. American Psychological Association, Washington, DC, pp73-106, 2007.

6. Costa PT Jr and McCrae RR: Personality disorders and the five-factor model of personality. J Pers Disord 4: 362-371, 1990.

7. Digman JM: Personality structure: Emergence of the five-factor model. Annu Rev Psychol 41: 417-440, 1990.

8. Bermúdez J: Personality and health-protective behavior. Eur J Pers 13: 83-103, 1999.
9. Clarke S and Robertson IT: A meta-analytic review of the big five personality factors and accident involvement in occupational and non-occupational settings. J Occup Organ Psychol 78: 355-376, 2005.

10. Vollrath $\mathrm{M}$ and Torgersen S: Who takes health risks? A probe into eight personality types. Pers Individ Dif 32: 1185-1198, 2002.

11. Castanier C, Scanff CL and Woodman T: Who takes risks in high-risk sports? A typological personality approach. Res Q Exerc Sport 81: 478-484, 2010.

12. Tulloch J and Lupton D: Risk and everyday life. SAGE Publications Ltd., London, 2003.

13. Lyng S: Edgework: The sociology of risk taking. Routledge, London, pp17-49, 2005.

14. Barlow M, Woodman T and Hardy L: Great expectations: different high-risk activities satisfy different motives. J Pers Soc Psychol 105: 458-475, 2013.

15. Hare OA, Wetherell MA and Smith MA: State anxiety and cortisol reactivity to skydiving in novice versus experienced skydivers. Physiol Behav 118: 40-44, 2013.

16. Bołdak A and Guszkowska M: State anxiety change after a parachute jump and its determinants: gender, experience, and temperament features. Biomed Hum Kinet 5: 113-120, 2013.

17. Breedlove SM and Hampson E: Sexual differentiation of the brain and behaviour. In: Behavioral Endocrinology. Becker J, Breedlove SM, Crews D and McCarthy MM (eds). 2nd edition. MIT Press, Cambridge, MA, pp75-114, 2002.

18. Tobet SA and Baum MJ: Role for prenatal estrogen in the development of masculine sexual behavior in the male ferret. Horm Behav 21: 419-429, 1987.

19. Ronay R and Von Hippel W: Power, testosterone, and risk-taking. J Behav Decis Mak 23: 473-482, 2010.

20. Boissy A, Bouissou M. Effects of androgen treatment on behavioural and physiological responses of heifers to fear-eliciting situations. Horm Behav 28: 66-83, 1994

21. Andrew RJ and Rogers LJ: Testosterone, search behaviour and persistence. Nature 237: 343-346, 1972.

22. Apicella CL, Dreber A, Campbell B, Gray P, Hoffman M and Little A: Testosterone and financial risk preferences. Evol Hum Behav 29: 384-390, 2008

23. Booth A, Johnson DR and Granger DA: Testosterone and men's health. J Behav Med 22: 1-19, 1999.

24. Salminen EK, Portin RI, Koskinen A, Helenius H and Nurmi M: Associations between serum testosterone fall and cognitive function in prostate cancer patients. Clin Cancer Res 10: 7575-7582, 2004

25. Irimia V: Physical Risk-Taking, Digit Ratio, and Circulating Testosterone (unpublished $\mathrm{PhD}$ thesis). Concordia University, 2017.

26. Cohen-Bendahan $\mathrm{CC}$, van de Beek $\mathrm{C}$ and Berenbaum SA: Prenatal sex hormone effects on child and adult sex-typed behavior: Methods and findings. Neurosci Biobehav Rev 29: 353-384, 2005.

27. Neyse L and Brañas-Garza P: Digit Ratio Measurement Guide. Kiel Institute for the World Economy, 2014.

28. Manning JT, Scutt D, Wilson J and Lewis-Jones DI: The ratio of 2nd to 4th digit length: A predictor of sperm numbers and concentrations of testosterone, luteinizing hormone and oestrogen. Hum Reprod 13: 3000-3004, 1998

29. Berenbaum SA, Bryk KK, Nowak N, Quigley CA and Moffat S: Fingers as a marker of prenatal androgen exposure. Endocrinology 150: 5119-5124, 2009.

30. Manning JT: Resolving the role of prenatal sex steroids in the development of digit ratio. Proc Natl Acad Sci USA 108: 16143-16144, 2011.

31. McIntyre MH: The use of digit ratios as markers for perinatal androgen action. Reprod Biol Endocrinol 4: 10, 2006.

32. Voracek M, Dressler SG and Manning JT: Evidence for assortative mating on digit ratio (2D:4D), a biomarker for prenatal androgen exposure. J Biosoc Sci 39: 599-612, 2007.

33. Fink B, Manning JT and Neave N: Second to fourth digit ratio and the 'big five'personality factors. Pers Individ Dif 37: 495-503, 2004.

34. Fink B, Neave N, Laughton K and Manning JT: Second to fourth digit ratio and sensation seeking. Pers Individ Dif 41: 1253-1262, 2006.

35. Coates JM, Gurnell M and Rustichini A: Second-to-fourth digit ratio predicts success among high-frequency financial traders. Proc Natl Acad Sci USA 106: 623-628, 2009.

36. Stenstrom E, Saad G, Nepomuceno MV and Mendenhall Z: Testosterone and domain-specific risk: Digit ratios (2D:4D and rel2) as predictors of recreational, financial, and social risk-taking behaviors. Pers Individ Dif 51: 412-416, 2011. 
37. Coco M, Perciavalle V, Maci T, Nicoletti F, Di Corrado D and Perciavalle V: The second-to-fourth digit ratio correlates with the rate of academic performance in medical school students. Mol Med Rep 4: 471-476, 2011.

38. Tester N and Campbell A: Sporting achievement: What is the contribution of digit ratio? J Pers 75: 663-677, 2007.

39. Manning JT: The ratio of 2 nd to 4 th digit length and performance in skiing. J Sports Med Phys Fitness 42: 446-450, 2002.

40. Manning JT and Taylor RP: Second to fourth digit ratio and male ability in sport: Implications for sexual selection in humans. Evol Hum Behav 22: 61-69, 2001.

41. Perciavalle V, Di Corrado D, Petralia MC, Gurrisi L, Massimino S and Coco M: The second-to-fourth digit ratio correlates with aggressive behavior in professional soccer players. Mol Med Rep 7: 1733-1738, 2013

42. Voracek M, Tran US and Dressler SG: Digit ratio (2D: 4D) and sensation seeking: New data and meta-analysis. Pers Individ Dif 48: 72-77, 2010.

43. Garbarino E, Slonim R and Sydnor J: Digit ratios (2D: 4D) as predictors of risky decision making for both sexes. J Risk Uncertain 42: 1-26, 2011

44. Caprara GV, Barbaranelli C, Borgogni L and Vecchione M: BFQ-2. Big Five Questionnaire 2. Giunti Organizzazioni Speciali, Firenze, 2008

45. Edwards AL and Edwards LK: Social desirability and Wiggin's MMPI content scales. J Pers Soc Psychol 62: 147-153, 1992.

46. Spielberger CD: Manual for the State -Trait Anxiety Inventory (STAI). Consulting Psychologists Press, Palo Alto, CA, 1983.

47. McNair D, Lorr M, and Droppleman LF: Manual for the Profile of Mood States. Educational and Industrial Testing Services, San Diego, CA, 1971.

48. Woodman T, Barlow M, Bandura C, Hill M, Kupciw D and Macgregor A: Not all risks are equal: The risk taking inventory for high-risk sports. J Sport Exerc Psychol 35: 479-492, 2013.

49. Kimura S, Schaumann BA, Plato CC and Kitagawa T: Embryological development and prevalence of digital flexion creases. Anat Rec 226: 249-257, 1990.

50. Manning JT, Churchill AJ and Peters M: The effects of sex, ethnicity, and sexual orientation on self-measured digit ratio (2D:4D). Arch Sex Behav 36: 223-233, 2007.

51. Williams TJ, Pepitone ME, Christensen SE, Cooke BM Huberman AD, Breedlove NJ, Breedlove TJ, Jordan CL and Breedlove SM: Finger-length ratios and sexual orientation. Nature 404: 455-456, 2000

52. Costa PT Jr and McCrae RR: NEO PI-R professional manual. Psychological Assessment Resources, Inc., Odessa, FL, 1992.

53. Van Vugt M and Hart CM: Social identity as social glue: the origins of group loyalty. J Pers Soc Psychol 86: 585-598, 2004.

54. Price IR and Bundesen C: Emotional changes in skydivers in relation to experience. Pers Individ Dif 38: 1203-1211, 2004

55. Lavallée L and Flint F: The relationship of stress, competitive anxiety, mood state, and social support to athletic injury. J Athl Train 31: 296-299, 1996.

56. Hirschfeld RM, Holzer C, Calabrese JR, Weissman M, Reed M, Davies M, Frye MA, Keck P, McElroy S, Lewis L, et al: Validity of the mood disorder questionnaire: A general population study. Am J Psychiatry 160: 178-180, 2003.

57. Thatcher J, Reeves S, Dorling D and Palmer A: Motivation, stress, and cortisol responses in skydiving. Percept Mot Skills 97: 995-1002, 2003

58. Petralia MC, Perciavalle V, Basile MS, Alagona G, Monaca A, Buscemi A and Coco M: The rise of lactic acid, from a pharmacist's laboratory to entry into the central nervous system. Sport Sci Health 14: 455-457, 2018

59. Coco M, Platania S, Castellano S, Sagone E, Ramaci T, Petralia MC, Agati M, Massimino S, Di Corrado D, Guarnera M, et al: Memory, personality and blood lactate during a judo competition. Sport Sci Health (In press)

60. Coco M, Alagona G, De Maria G, Rapisarda G, Costanzo E, Perciavalle V and Perciavalle V: Relationship of high blood lactate levels with latency of visual-evoked potentials. Neurol Sci 36: 541-546, 2015
61. Coco M, Fiore AS, Perciavalle V, Maci T, Petralia MC and Perciavalle V: Stress exposure and postural control in young females. Mol Med Rep 11: 2135-2140, 2015.

62. Coco M, Alagona G, Perciavalle V, Rapisarda G, Costanzo E and Perciavalle V: Brainstem excitability is not influenced by blood lactate levels. Somatosens Mot Res 30: 90-95, 2013.

63. Perciavalle V, Alagona G, De Maria G, Rapisarda G, Costanzo E, Perciavalle V and Coco M: Somatosensory evoked potentials and blood lactate levels. Neurol Sci 36: 1597-1601, 2015.

64. Perciavalle V, Di Corrado D, Scuto C, Perciavalle V and Coco M: Attention and blood lactate levels in equestrians performing show jumping. Percept Mot Skills 118: 733-745, 2014.

65. Perciavalle V, Marchetta NS, Giustiniani S, Borbone C, Perciavalle V, Petralia MC, Buscemi A and Coco M: Attentive processes, blood lactate and CrossFit ${ }^{\circledR}$. Phys Sportsmed 44: 403-406, 2016.

66. Coco M: The brain behaves as a muscle? Neurol Sci 38: $1865-1868,2017$

67. Coco M, Alagona G, Perciavalle V, Perciavalle V, Cavallari P and Caronni A: Changes in cortical excitability and blood lactate after a fatiguing hand-grip exercise. Somatosens Mot Res 31: 35-39, 2014.

68. Perciavalle V, Di Corrado D, Scuto C, Perciavalle V and Coco M: Anthropometrics related to the performance of a sample of male swimmers. Percept Mot Skills 118: 940-950, 2014.

69. Aspara J and Van Den Bergh B: Naturally designed for masculinity vs. femininity? Prenatal testosterone predicts male consumers' choices of gender-imaged products. Int J Res Mark 31: 117-121, 2014

70. Lens I, Driesmans K, Pandelaere M and Janssens K: Would male conspicuous consumption capture the female eye? Menstrual cycle effects on women's attention to status products. J Exp Soc Psychol 48: 346-349, 2012.

71. Saad G and Stenstrom E: Calories, beauty, and ovulation: The effects of the menstrual cycle on food and appearance-related consumption. J Consum Psychol 22: 102-113, 2012.

72. Durante KM, Griskevicius V, Hill SE, Perilloux C and Li NP: Ovulation, female competition, and product choice: Hormonal influences on consumer behavior. J Consum Res 37: 921-934, 2010.

73. Coco M, Alagona G, Perciavalle V, Perciavalle V, Cavallari P and Caronni A: Changes in cortical excitability and blood lactate after a fatiguing hand-grip exercise. Somatosens Mot Res 31: 35-39, 2014.

74. Fornaro M, De Berardis D, De Pasquale C, Indelicato L, Pollice R, Valchera A, Perna G, Iasevoli F, Tomasetti C, Martinotti G, et al: Prevalence and clinical features associated to bipolar disorder-migraine comorbidity: A systematic review. Compr Psychiatry 56: 1-16, 2015

75. Conti D, Di Nuovo S, Cangelosi A and Di Nuovo A: Lateral specialization in unilateral spatial neglect: A cognitive robotics model. Cogn Process 17: 321-328, 2016.

76. Conti D, Di Nuovo A, Cirasa C and Di Nuovo S: A comparison of kindergarten storytelling by human and humanoid robot with different social behavior. In: HRI '17. Proceedings of the Companion of the 2017 ACM/IEEE International Conference on Human-Robot Interaction - HRI '17. ACM, New York, NY, pp97-98, 2017.

77. Petralia MC, Coco M, Maci T, Minissale C, De Pasquale C, D'orto G, Basile MS, Donato E, Maniaci M, Commodari E, et al: Successfully aging. Choice of life or life that choices? Acta Med Mediter 34: 107, 2018.

78. Saad G and Vongas JG: The effect of conspicuous consumption on men's testosterone levels. Organ Behav Hum Decis Process 110: 80-92, 2009. 